\title{
SHRINKING CERTAIN SLICED DECOMPOSITIONS OF $E^{n+1}$
}

\author{
ROBERT J. DAVERMAN ${ }^{1}$ AND D. KRISS PRESTON
}

\begin{abstract}
We set forth a connection, based on relatively elementary techniques, between the shrinkability of product decompositions of $E^{n+1}$ and that of sliced decompositions. In particular, if $G$ is a decomposition of $E^{n+1}$ such that each decomposition element $g$ is contained in some horizontal slice $E^{n} \times\{s\}$ and if the decomposition $G^{s}$ of $E^{n}$, consisting of those subsets $g$ of $E^{n}$ for which $g \times\{s\} \in$ $G$, expands to a shrinkable decomposition $G^{s} \times E^{1}$ of $E^{n} \times E^{1}$, we show then that $G$ itself is shrinkable.
\end{abstract}

1. Introduction. We say that a decomposition $G$ of $X \times E^{1}$ (or of $X \times S^{1}$ ) is sliced if each decomposition element $g$ of $G$ is contained in some slice $X \times\{s\}$, where $s \in E^{1}$ (or $s \in S^{1}$ ). Product decompositions, which arise from a decomposition $G^{\prime}$ of $X$ by defining $G$ to be the decomposition of $X \times E^{1}$ expressed simply as $G=G^{\prime} \times E^{1}$ and composed of all elements $g^{\prime} \times\{s\}, g^{\prime} \in G^{\prime}$ and $s \in E^{1}$, serve as a natural and important class of sliced decompositions. Moreover, at least for $n \geqslant 4$, there are strong results concerning the shrinkability of product decompositions of $E^{n} \times E^{1}$; the archetype, from our point of view, asserts that, for any cell-like decomposition $G^{\prime}$ of $E^{n}(n \geqslant 4)$ such that the closure of the image of its nondegenerate elements in the associated decomposition space is $(n-2)$ dimensional, the product decomposition $G=G^{\prime} \times E^{1}$ of $E^{n+1}=E^{n} \times E^{1}$ is shrinkable, implying that $E^{n+1} / G$ is topologically $E^{n+1}$ [C, Theorem 10.1], [Ed].

There has been comparatively little study of sliced decompositions. We regard this as a serious oversight, which we attempt to correct in this paper. We show that not only do such decompositions act as a useful aid in investigations of significant decomposition problems, but also they form a surprisingly strong alliance with product decompositions. An evidence for this alliance is a corollary to our theorem, establishing that if $G$ is a sliced decomposition of $E^{n+1}$ such that the decomposition $G^{s}$ of $E^{n}$ induced by any slice $E^{n} \times\{s\}$, consisting of those subsets $g$ of $E^{n}$ for which $g \times\{s\} \in G$, expands to a shrinkable product decomposition $G^{s} \times E^{1}$ of $E^{n+1}$, then the sliced decomposition $G$ itself is shrinkable.

2. Notation. Given a decomposition $G$, which we understand throughout to be an upper semicontinuous one, we use $H_{G}$ to denote its set of nondegenerate elements and $N_{G}$ to denote the union of these nongenerate elements.

Received by the editors January 18, 1979 and, in revised form, August 6, 1979.

AMS (MOS) subject classifications (1970). Primary 54B15, 57A15; Secondary 57A60, 57A10, 54 F65.

Key words and phrases. Upper semicontinuous decomposition, cell-like, shrinkable decomposition, shrinkability criterion, sliced decomposition, embedding dimension.

${ }^{1}$ Supported in part by National Science Foundation Grant MCS 76-07274. 
Given a decomposition $G$ of a space $X$, we say that $G$ is $k$-dimensional if, under the natural decomposition map $\pi: X \rightarrow X / G$, the dimension of $\pi\left(N_{G}\right)$ is $k$, and that $G$ is closed $k$-dimensional if the closure of $\pi\left(N_{G}\right)$ has dimension $k$.

In studying a sliced decomposition $G$ of $S^{n} \times S^{1}$, for each slice $S^{n} \times\{s\}$ one has two distinct decompositions associated with that slice: one a decomposition $G^{s}$ of $S^{n}$ consisting of all sets $g^{\prime}$ in $S^{n}$ such that $g^{\prime} \times\{s\} \in G$, and the other a decomposition $G(s)$ of $S^{n} \times S^{1}$ consisting of all sets $g$ of $G$ that are contained in the slice $S^{n} \times\{s\}$ together with the singletons of $S^{n} \times\left(S^{1}-\{s\}\right)$. The latter decomposition $G(s)$ coincides with the trivial extension of the decomposition $G^{s} \times\{s\}$ of $S^{n} \times\{s\}$ to all of $S^{n} \times S^{1}$.

All decompositions encountered here are cell-like, abbreviated as $\mathrm{CE}$, which means that the elements of the decomposition are cell-like sets. The perennial question about such decompositions is their shrinkability. A decomposition $G$ of a locally compact metric space $X$ (into compact subsets) is said to be shrinkable if for each $\varepsilon>0$ there exists a homeomorphism $h$ of $X$ onto itself such that:

distance $(\pi(X), \pi h(x))<\varepsilon$ for each $x \in X$ (where $\pi$ denotes the decomposition $\operatorname{map} X \rightarrow X / G)$,

$\operatorname{diam} h(g)<\varepsilon$.

One phrasing of the classical Bing Shrinkability Criterion (see [B1], [B2]) states that a decomposition $G$ of a locally compact (or, more generally, a complete [MV]) metric space $X$ is shrinkable if and only if the decomposition map $\pi: X \rightarrow X / G$ can be approximated by homeomorphisms.

\section{The main result.}

Lemma 1. Suppose that $G$ is a sliced $C E$ decomposition of $S^{n} \times S^{1}$ and that $C$ is a compact 0 -dimensional subset of $S^{1}$ satisfying

(1) $N_{G}$ is contained in $S^{n} \times C$, and

(2) for each $c \in C$ the decomposition $G(c)$ is shrinkable.

Then $G$ itself is shrinkable.

Proof. Fix a metric $\rho$ on $\left(S^{n} \times S^{1}\right) / G$, and determine $\delta \in(0, \varepsilon / 3)$ so that if $F$ : $S^{n} \times S^{1} \rightarrow S^{n} \times S^{1}$ is a homeomorphism moving points less than $\delta$, then $\rho(\pi, \pi F)$ $<\varepsilon / 3$.

It follows either from the local contractibility of the homeomorphism group of $S^{n} \times S^{1}$ [D3] or, as long as $n \neq 3$, from the Armentrout-Siebenmann Approximation Theorem [A], [S], that a decomposition $G$ of $S^{n} \times S^{1}$ is shrinkable if and only if, given a neighborhood $U$ of $\mathrm{Cl} N_{G}$, one can find homeomorphisms $h$ shrinking $G$, in the usual sense, while keeping points of $\left(S^{n} \times S^{1}\right)-U$ fixed. In particular, as a consequence of hypothesis (2), for each $c \in C$ there exists a homeomorphism $h_{c}$ of $S^{n} \times S^{1}$ to itself shrinking each $g \in G(c)$ to diameter less than $\varepsilon / 3$, satisfying $\rho\left(\pi, \pi h_{c}\right)<\varepsilon / 3$, and fixing points outside $S^{n} \times U_{c}$, where $U_{c}$ denotes the $\delta$-neighborhood of $c$ in $S^{1}$.

Corresponding to each $c \in C$ there is an open interval $J_{c}$ such that $c \in J_{c} \subset U_{c}$ and, for each $g \in G \cap\left(S^{n} \times J_{c}\right)$, the diameter of $h_{c}(g)$ is less than $\varepsilon / 3$. From the open cover $\left\{J_{c} \mid c \in C\right\}$ we extract a finite subcover $\left\{J_{c(i)} \mid i=1, \ldots, N\right\}$, and we 
cut back these intervals slightly so that the collection consists of pairwise disjoint intervals.

For $i=1, \ldots, N$ we name a homeomorphism $f_{i}$ of $\mathrm{Cl} U_{c(i)}$ onto $\mathrm{Cl} J_{c(i)}$ that keeps a neighborhood of $J_{c(i)} \cap C$ pointwise fixed (preventing it from interchanging the ends of the intervals), and then name the product homeomorphism $F_{i}=1 \times f_{i}$ : $S^{n} \times S^{1} \rightarrow S^{n} \times S^{1}$. Finally, we produce the required shrinking homeomorphism $h$ as the one equal to $F_{i} h_{c(i)} F_{i}^{-1}$ on $S^{n} \times J_{c(i)}(i=1, \ldots, N)$ and equal to the identity elsewhere.

LEMMA 2. Let $G$ be a decomposition of $S^{n}$ such that $G \times S^{1}$ is a shrinkable decomposition of $S^{n} \times S^{1}, C$ a compact subset of $S^{1}$, and $\theta$ a map of $S^{n} \times S^{1}$ to itself realizing the decomposition $G \times C$, trivially extended over $S^{n} \times S^{1}$ (that is, each nondegenerate inverse set $\theta^{-1}(x)$ equals $g \times\{c\}$ for some $g \in G$ and $\left.c \in C\right)$. Then for each point $c$ of $C, \theta\left(S^{n} \times\{c\}\right)$ is bicollared in $S^{n} \times S^{1}$.

Proof. Consider any component $U$ of $S^{1}-C$. The map $\theta$ is $1-1$ on $S^{n} \times U$ and crushes to points those elements of $G$ in $S^{n} \times \mathrm{Bd} U$. By the hypothesis, slightly modified, $\theta\left(\left(G \times S^{1}\right) \cap\left(S^{n} \times U\right)\right)$ is a shrinkable decomposition of $\theta\left(S^{n} \times U\right)$, the diameters of whose elements approach zero near the frontier of $\theta\left(S^{n} \times U\right)$. As a result, there is a shrinking of this portion of $\theta\left(G \times S^{1}\right)$ to small size that keeps points fixed outside $\theta\left(S^{n} \times U\right)$. Among the (at most) countably many sets of the form $\theta\left(S^{n} \times U\right)$, only a finite number contain an element from $\theta\left(G \times S^{1}\right)$ with diameter greater than any preassigned positive number. Thus, a careful shrinking, as described above, of finitely many portions squeezes all of $\theta\left(G \times S^{1}\right)$ to small size, keeping points of $\theta\left(S^{n} \times C\right)$ fixed. As a result, in the usual limiting fashion one can produce a map $\theta^{\prime}$ of $S^{n} \times S^{\prime}$ to itself fixing the points of $\theta\left(S^{n} \times C\right)$ and realizing the decomposition $\theta\left(G \times S^{1}\right)$. In other words, each nondegenerate inverse $\left(\theta^{\prime}\right)^{-1}(x)$ equals $\theta(g \times\{s\})$ for some $g \in G$ and $s \in S^{1}-C$.

Let $\pi: S^{n} \times S^{1} \rightarrow\left(S^{n} \times S^{1}\right) /\left(G \times S^{1}\right)$ denote the decomposition map. Then $\theta^{\prime} \theta \pi^{-1}$ is a homeomorphism of $\left(S^{n} \times S^{1}\right) /\left(G \times S^{1}\right)$, which is naturally homeomorphic to $\left(S^{n} / G\right) \times S^{1}$, onto $S^{n} \times S^{1}$, implicitly carrying $\left(S^{n} / G\right) \times\{c\}$ onto $\theta^{\prime} \theta\left(S^{n} \times\{c\}\right)=\theta\left(S^{n} \times\{c\}\right)$, as required.

The idea central to the following argument is not entirely new; similar ideas, directed toward technically finer ends, can be discerned in papers by Woodruff [W] and by Cannon and Daverman [CD].

THEOREM. Suppose $G$ is a sliced CE decomposition of $S^{n} \times S^{1}$ satisfying

(1) for each $s \in S^{1}$ the decomposition $G(s)$ of $S^{n} \times S^{1}$ is shrinkable, and

(2) $S^{1}$ contains a countable dense set $D=\{d(i)\}$ for which the decompositions $G^{d(i)}$ of $S^{n}$ yield an $(n+1)$-manifold factor (that is, $G^{d(i)} \times E^{1}$ is shrinkable).

Then $G$ itself is shrinkable.

Proof. Reproducing the model of a monotone decomposition of $S^{1}$ with nondegenerate elements dense in $S^{1}$, we construct a CE map $f$ of $S^{1}$ to itself such that $f^{-1}(d(i))$ is an interval for each $d(i) \in D$ and that otherwise $f^{-1}(s)$ is a point. (Recall that any two countable dense subsets of $S^{1}$ are equivalently embedded there.) We name the product map $F=1 \times f$ of $S^{n} \times S^{1}$ to itself and consider the 
induced decomposition $G_{F}=\left\{F^{-1}(g) \mid g \in G\right\}$, which closely resembles $G$ except that elements from the $D$-levels have been stretched out along the vertical, or the $S^{1}$, direction.

Our intention is to prove that $G_{F}$ is shrinkable. Before proceeding with that, we point out how to establish the shrinkability of $G$, assuming the shrinkability of $G_{F}$, based on the diagram below:

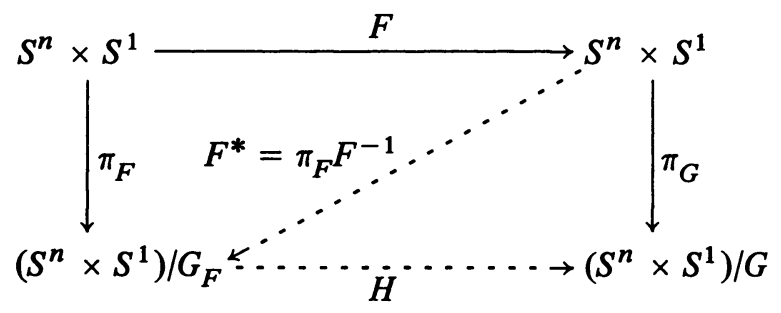

The natural function $H=\pi F\left(\pi_{F}\right)^{-1}$ clearly is a homeomorphism. The map $F$ clearly is approximable by homeomorphism and so also is $\pi_{F}$ (by assumption). As relatively easy consequences, one can show, in order, that $F^{*}=\pi_{F} F^{-1}$ and $\pi=H F^{*}$ are approximable by homeomorphism as well.

There are two related decompositions instrumental to the shrinking of $G_{F}$. The first of these is a level stratification, or a new sliced decomposition, defined as

$$
G_{1}=\left\{g_{F} \cap\left(S^{n} \times\{s\}\right) \mid g_{F} \in G_{F} \text { and } s \in S^{1}\right\} .
$$

The second is a fenestration of the first, giving us room to work, eliminating nondegenerate elements from a dense and open subset of levels. Specifically, for the union $N_{f}$ of the nondegenerate elements of $\left\{f^{-1}(s) \mid s \in S^{1}\right\}, G_{2}$ consists of the singletons from $S^{n} \times$ Int $N_{f}$ together with $\left\{g_{F} \cap\left(S^{n} \times\{s\}\right) \mid g_{F} \in G_{F}\right.$ and $s \in$ $\left.\mathrm{Cl}\left(S^{1}-N_{f}\right)\right\}$. In other words, $G_{2}$ has for its nondegenerate elements those (nondegenerate) elements of $G_{1}$ from levels not interior to any nondegenerate element of $f$.

According to Lemma 1 , the fenestrated decomposition $G_{2}$ is shrinkable. Hence, there exists a map $\theta_{2}$ of $S^{n} \times S^{1}$ to itself that realizes this decomposition, in the sense that $G_{2}=\left\{\theta_{2}^{-1}(x) \mid x \in S^{n} \times S^{1}\right\}$, and where $\theta_{2}$ is the end of a pseudo-isotopy $\psi_{t}^{2}$ defined on $S^{n} \times S^{1}$ such that $\rho\left(\pi_{F}, \pi_{F} \psi_{t}^{2}\right)<\varepsilon / 3$.

Naturally, we next look at the modified decomposition $\theta_{2}\left(G_{1}\right)$, whose nondegenerate elements are partitioned into countably many (curvilinear) product decompositions. Explicitly, for each inverse $A_{i}=f^{-1}(d(i))$ in $S^{1}, \theta_{2}\left(G_{1} \cap\left(S^{n} \times \operatorname{Int} A_{i}\right)\right)$ is topologically equivalent with $G^{d(i)} \times E^{1}$, which is shrinkable by hypothesis. Exactly as in the proof of Lemma 2, we show that $\theta_{2}\left(G_{1}\right)$ is shrinkable. As before, then, there exists a map $\theta_{1}$ of $S^{n} \times S^{1}$ to itself that realizes $\theta_{2}\left(G_{1}\right)$, where $\theta_{1}$ is the end of a pseudo-isotopy $\psi_{t}^{1}$ such that $\rho\left(\pi, \pi \psi_{t}^{1}\right)<\varepsilon / 3$.

Finally, we turn to the resultant decomposition $\theta_{1} \theta_{2}\left(G_{F}\right)$, from which all the strata of $G_{1}$ have been crushed to points, leaving only products of an arc with the decomposition spaces $S^{n} / G^{d(i)}$ associated with the dense set $D$ of special levels. The fiber arcs of these products must be shrunk. In the situation at hand this presents no difficutly because (1) the maximal diameter $\lambda_{i}$ of a fiber arc from the product space associated with $S^{n} / G^{d(i)}$ approaches zero as $i$ increases and (2) each 
product space is collared, from both boundary components (see Lemma 2). Then the required shrinking is a much simpler effort than those described in [D1, §5] or in [W, Theorem 2]. It can also be achieved readily by parameterizing the techniques for shrinking a null sequence of tame arcs from $S^{n}$ [B2, Theorem 1]. Again there exists a map $\theta_{F}$ realizing the decomposition $\theta_{1} \theta_{2}\left(G_{F}\right)$, where $\theta_{F}$ is the end of a pseudo-isotopy $\psi_{t}^{F}$ such that $\rho\left(\pi_{F}, \pi_{F} \psi_{t}^{F}\right)<\varepsilon / 3$.

Now we see that $\theta_{F} \theta_{1} \theta_{2}$ realizes the decomposition $G_{F}$. Choosing $\gamma \in[0,1)$ very close to 1, we produce a homeomorphism $\psi_{\gamma} \psi_{\gamma}^{1} \psi_{\gamma}^{2}$ of $S^{n} \times S^{1}$ to itself fulfilling the conditions required to verify that $G_{F}$ is shrinkable.

REMARK. The compactness of the domain $S^{n} \times S^{1}$ considerably simplifies the proofs, but is not necessary for them. In the applications given in the next section we transfer the setting from the compact $S^{n} \times S^{1}$ to the more natural $E^{n} \times E^{1}$.

\section{Applications.}

COROllary 0. If $G$ is a sliced decomposition of $E^{n} \times E^{1}$ so that, for each $s \in E^{1}$, $G^{s} \times E^{1}$ is shrinkable, then $G$ is shrinkable.

Proof. Since $G^{s} \times E^{1}$ is shrinkable, Daverman [D3] shows that $G(s)$ is shrinkable.

Next we obtain another proof to a result of Dyer and Hamstrom [DH].

Corollary 1. Every sliced decomposition $G$ of $E^{3}$ is shrinkable.

Proof. It is a classical result, in its original form due to R. L. Moore [M], that each CE decomposition of $E^{2}$ is shrinkable. One can easily see that its product with $E^{1}$ is also shrinkable.

We also obtain another proof for a result of Everett [Ev, Theorem 1].

COROllaRY 2. If $G$ is a 0-dimensional $C E$ decomposition of $E^{n}$, considered as $E^{n} \times\{0\}$ in $E^{n} \times E^{1}$, then the trivial extension $G^{\prime}$ (consisting of elements of $G$ and singletons from $\left.E^{n} \times\left(E^{1}-\{0\}\right)\right)$ to all of $E^{n+1}$ is shrinkable.

Proof. By elementary methods like those of [HW, Chapter V], there exists a map $f: E^{n} / G \rightarrow E^{1}$ such that $f \mid \pi\left(N_{G}\right)$ is an embedding. Let $\theta$ denote the $E^{n}$-coordinate preserving homeomorphism of $E^{n+1}$ to itself defined by $\langle x, s\rangle \rightarrow\langle x, s+f \pi(x)\rangle$. Then $\theta(G)$ is a sliced CE decomposition of $E^{n+1}$ such that each slice $E^{n} \times\{s\}$ contains at most one nondegenerate element $\theta(g)$. Since each such $\theta(g)$ is itself cellular in $E^{n+1}$ (see [CM]), each $(\theta(G))(s)$ is shrinkable. Furthermore, for $s$ from a dense subset of $E^{1},(\theta(G))^{s}$ contains only singletons, trivially implying that its product with $E^{1}$ is shrinkable.

Corollary 3. Suppose that $G$ is a sliced $C E$ decomposition of $E^{n+1}(n>4)$ such that $\left(E^{n+1} / G\right)$ is a finite dimensional space and that $E^{1}$ contains a dense subset $D$ for which the decompositions

$$
G^{d}=\left\{g \subset E^{n} \mid g \times d \in G\right\} \quad(d \in D)
$$

yield $E^{n+1}$ factors (that is, $G^{d} \times E^{1}$ is shrinkable). Then $G$ itself is shrinkable. 
Proof. Daverman [D2] uses the Approximation Theorem of Edwards [Ed] to show that, for each $s \in E^{1}$, the decomposition $G(s)$ is shrinkable.

COROllary 4. If $G$ is a sliced $C E$ decomposition of $E^{n+1}(n>4)$ such that, for each $s \in E^{1}$, the decomposition $G(s)$ is closed $(n-2)$-dimensional, then $G$ is shrinkable.

Proof. The combination of a result by Cannon [C, Theorem 10.1] and Edwards' Approximation Theorem [Ed] implies that each $G^{s} \times E^{1}$ is shrinkable.

COROLlaRY 5. If $G$ is a sliced CE decomposition of $E^{n+1}(n>4)$ such that, for each $s \in E^{1}$, the decomposition $G(s)$ is $(n-3)$-dimensional, then $G$ is shrinkable.

Proof. Daverman [D2] shows that each $G^{s} \times E^{1}$ is shrinkable.

COROLlaRY 6. Closed ( $n-2)$-dimensional sliced CE decompositions and $(n-3)$ dimensional sliced $C E$ decompositions of $E^{n+1}(n>4)$ are shrinkable.

COROLlaRY 7. If $G$ is a sliced $C E$ decomposition of $E^{4}$ such that, for each $s \in E^{1}$, $G(s)$ is 0 -dimensional, then $G$ is shrinkable.

Proof. By Corollary 2, $G(s)$ is shrinkable, and by [DR, Corollary $1 \mathrm{~A}], G^{s} \times E^{1}$ is shrinkable.

Corollary 8. If $G$ is a 1-dimensional $C E$ decomposition of $E^{3}$, considered as $E^{3} \times\{0\}$ in $E^{3} \times E^{1}$, then the trivial extension $G^{\prime}$ to all of $E^{4}$ is shrinkable.

Proof. Much like the argument for Corollary 2, we construct a homeomorphism $\theta$ of $E^{4}$ to itself such that the equivalent decomposition $\theta\left(G^{\prime}\right)$ is sliced and on each slice the restricted decomposition $\left(\theta\left(G^{\prime}\right)\right)(s)$ is 0 -dimensional. The result follows from Corollary 7.

Corollary 9. If $G$ is a sliced $C E$ decomposition of $E^{4}$ such that, for each $s \in E^{1}$, the decomposition $G(s)$ is either 0-dimensional or $N_{G(s)}$ has embedding dimension no more than 1 , then $G$ is shrinkable.

Proof. Here there is an important decomposition-theoretic detail at work: for $s \in E^{1}, N_{G(s)}$ has embedding dimension 1 and, therefore, is 1-dimensional; it follows from [K] that its image in $E^{4} / G(s)$ also is 1-dimensional. By Corollary 8, $G(s)$ is shrinkable, and by either [DR] or [DP], $G^{s} \times E^{1}$ is shrinkable as well.

COROLlaRY 10. If $G$ is a sliced $C E$ decomposition of $E^{n} \times E^{1}$ so that each slice is a product decomposition of $E^{n-1} \times E^{1}=E^{n}$ and $E^{n+1} / G$ is finite dimensional, then $G$ is shrinkable.

Proof. By Corollary 0, we need only show that each slice is an $E^{n+1}$-factor. But since each slice is already a product with $E^{1}$, Corollary 10 follows from the result of [D2] that the product of $E^{2}$ and any cell-like decomposition of $E^{n-1}$ (with finite dimensional image) is shrinkable.

In keeping with our belief that sliced decomposition theory must parallel product decomposition theory we present the next corollary, which generalizes Daverman's [D2] result concerning products with $E^{2}$. 
Corollary 11. If $G$ is a twice-sliced $C E$ decomposition of $E^{n} \times E^{2}$, in the sense that each $g \in G$ lies in some slice $E^{n} \times\left\{s_{g}\right\}, s_{g} \in E^{2}$, and if $E^{n+2} / G$ is finite dimensional, then $G$ is shrinkable.

Proof. It is helpful to view $G$ as a decomposition of $E^{n} \times E^{1} \times E^{1}$ sliced in each $E^{1}$ direction. Corollary 0 implies that with respect to one of the $E^{1}$ directions, $G$ may as well be a product. Now Corollary 10 finishes the proof.

\section{REFERENCES}

A. S. Armentrout, Cellular decompositions of 3-manifolds that yield 3-manifolds, Mem. Amer. Math. Soc. No. 107 (1971).

B1. R. H. Bing, A homeomorphism between the 3-sphere and the sum of two solid horned spheres, Ann. of Math. (2) 56 (1952), 354-362.

B2. $\longrightarrow$ Upper semicontimuous decompositions of $E^{3}$, Ann. of Math. (2) 65 (1957), 363-374.

C. J. W. Cannon, Shrinking cell-like decompositions of manifolds. Codimension three, Ann. of Math. (to appear).

CD. J. W. Cannon and R. J. Daverman, A totally wild flow (to appear).

CM. M. L. Curtis and D. R. McMillan, Cellularity of sets in products, Michigan Math. J. 9 (1962), 299-302.

D1. R. J. Daverman, Every crumpled n-cube is a closed n-cell-complement, Michigan Math. J. 24 (1977), 225-241.

D2. , Detecting the disjoint discs property 254 (1979), 217-236.

D3. __ Applications of local contractibility of manifold homeomorphism groups (to appear).

DP. R. J. Daverman and D. K. Preston, Cell-like 1-dimensional decompositions of $S^{3}$ are 4 -manifold factors (to appear).

DR. R. J. Daverman and W. H. Row, Cell-like 0-dimensional decompasitions of $S^{3}$ are 4-manifold factors, Trans. Amer. Math. Soc. 254 (1979), 217-236.

DH. E. Dyer and M. E. Hamstrom, Completely regular mappings, Fund. Math. 45 (1958), 103-118.

Ed. R. D. Edwards, Approximating certain cell-like maps by homeomorphisms, manuscript. See Notices Amer. Math. Soc. 24 (1977), p. A-649, Abstract 751-G5.

EK. R. D. Edwards and R. C. Kirby, Deformations of spaces of embeddings, Ann. of Math. (2) 93 (1971), 63-88.

Ev. D. L. Everett, Embedding theorems for decomposition spaces, Houston J. Math. 3 (1977), 351-368.

HW. W. Hurewicz and H. Wallman, Dimension theory, Princeton Univ. Press, Princeton, N. J., 1948.

K. G. Kozlowski, Images of ANR's, Trans. Amer. Math. Soc. (to appear).

MV. A. Marin and Y. M. Visetti, A general proof of Bing's Shrinkability Criterion, Proc. Amer. Math. Soc. 53 (1975), 501-507.

M. R. L. Moore, Concerning upper semi-continuous collections of continua, Trans. Amer. Math. Soc. 27 (1925), 416-428.

S. L. C. Siebenmann, Approximating cellular maps by homeomorphisms, Topology 11 (1972), 271-294.

W. E. P. Woodruff, Decomposition spaces having arbitrarily small neighborhoods with 2-sphere boundaries, Trans. Amer. Math. Soc. 232 (1977), 195-204.

Department of Mathematics, University of Tennessee, Knoxville, Tennesseb 37916 (current address of Robert J. Daverman)

Current address (D. Kriss Preston): Department of Mathematics, Hartwick College, Oneonta, New York 13820 